\title{
Regional Heterogeneity and an Unexpectedly High Abundance of Cooperia Punctata in Beef Cattle at Northern Latitude Were Revealed by ITS-2 rDNA Nemabiome Metabarcoding
}

\section{Eranga Lakshitha De Seram}

University of Saskatchewan Western College of Veterinary Medicine https://orcid.org/0000-0002-47726584

\section{Elizabeth Mary Redman}

University of Calgary - Faculty of Veterinary Medicine

\section{Felicity Kaye Wills}

University of Saskatchewan Western College of Veterinary Medicine

Camila de Queiroz

University of Calgary - Faculty of Veterinary Medicine

John Ross Campbell

University of Saskatchewan Western College of Veterinary Medicine

Cheryl Lynne Waldner

University of Saskatchewan Western College of Veterinary Medicine

\section{Sarah Elizabeth Parker}

University of Saskatchewan Western College of Veterinary Medicine

\section{Russell William Avramenko}

University of Calgary - Faculty of Veterinary Medicine

John Stuart Gilleard

University of Calgary - Faculty of Veterinary Medicine

Fabienne Dominique Uehlinger ( $\sim$ f.uehlinger@usask.ca )

1University of Saskatchewan

\section{Research}

Keywords: Beef herds, Cattle gastrointestinal nematodes, Cooperia, C. punctata, ITS-2 rDNA, Nemabiome metabarcoding, Northern latitudes

Posted Date: October 5th, 2021

DOl: https://doi.org/10.21203/rs.3.rs-951321/v1 
License: (c) (i) This work is licensed under a Creative Commons Attribution 4.0 International License. Read Full License

Version of Record: A version of this preprint was published at Parasites \& Vectors on January 6th, 2022. See the published version at https://doi.org/10.1186/s13071-021-05137-y. 


\section{Abstract}

\section{Background}

The species composition of cattle gastrointestinal nematode (GIN) communities can vary greatly between regions. Despite this, there is remarkably little large-scale surveillance data for cattle GIN species which is due, at least in part, to a lack of scalable diagnostic tools. This lack of regional GIN species-level data represents a major knowledge gap for evidence-based parasite management and assessing the status and impact of factors such as climate change and anthelmintic drug resistance.

\section{Methods}

This paper presents a large-scale survey of GIN in beef herds across western Canada using ITS-2 rDNA nemabiome metabarcoding. Individual fecal samples were collected from 6-20 randomly selected heifers $(n=1665)$ from each of 85 herds between September 2016 and February 2017 and 10-25 first season calves $(n=824)$ from each of 42 herds between November 2016 and February 2017.

\section{Results}

Gastrointestinal nematode communities in heifers and calves were similar in Alberta and Saskatchewan, with Ostertagia ostertagi and Cooperia oncophora being the predominant GIN species in all herds consistent with previous studies. However, in Manitoba, Cooperia punctata was the most predominant species overall and the most abundant GIN species in calves from 4/8 beef herds.

\section{Conclusions}

This study revealed a marked regional heterogenicity of GIN species in grazing beef herds in western Canada. The predominance of $C$. punctata in Manitoba is unexpected as although this parasite is often the most predominant cattle GIN species in more southerly latitudes, it is generally only a minor component of cattle GIN communities in northern temperate regions. We hypothesize that the unexpected predominance of $C$. punctata at such a northerly latitude represents a range expansion, likely associated with changes in climate, anthelmintic use, management, and/or animal movement. Whatever the cause, these results are of practical concern since $C$. punctata is more pathogenic than $C$. oncophora, the Cooperia species that typically predominates in cooler temperate regions. Finally, this study illustrates the value of ITS-2 rDNA nemabiome metabarcoding as a surveillance tool for ruminant GIN parasites.

\section{Background}

Co-infection with multiple gastrointestinal nematodes (GIN) species is common in cattle. The infection intensities of the different GIN species present will significantly influence the production impacts and the risk of clinical disease $[1,2]$. The relative abundance of different GIN species is known to vary greatly at the macro-geographical level, with the most abundant species in northern temperature regions being distinctly different from those at more southerly latitudes [3]. Many factors can affect GIN species 
distribution and abundance, including climate, grazing management, and anthelmintic use [4-8]. However, there is minimal regional GIN species-level data in cattle from most parts of the world. This important knowledge gap is most likely due to the laborious and specialized nature of identifying GIN species in fecal samples using coproculture and microscopy. The recent development of ITS-2 rDNA nemabiome metabarcoding provides a more accurate, objective, and scalable approach to examining GIN species composition of GIN parasite communities in grazing livestock $[3,9,10,11]$.

Previous work has shown that the two most abundant GIN species in cattle in Northern temperate regions in North America, including western Canada, are Ostertagia ostertagi and Cooperia oncophora, with a number of other species being present at variable but typically low levels [3,12]. Ostertagia ostertagi is the most pathogenic of the cattle GIN with the potential to cause severe clinical disease, but subclinical infections are more common at northern latitudes resulting in production loss such as reduced average daily gain and carcass quality $[1,2,13]$. Cooperia oncophora is less pathogenic but has become more concerned in recent years due to the high prevalence of macrocyclic lactone resistance $[8,14]$. Cooperia punctata, the most pathogenic of the bovine Cooperia species, is typically found at high levels in more southerly regions but was recently reported in several beef cattle herds in eastern Canada, albeit as a minority of the GIN species overall [3].

In this paper, we report the application of ITS-2 nemabiome metabarcoding to survey the GIN communities in 85 beef herds across western Canada. We found an unexpectedly high relative abundance of $C$. punctata in Manitoba, revealing a distinct regional heterogeneity in GIN communities of cattle reared under relatively similar management and climatic conditions. The occurrence of $C$. punctata beyond what is generally considered its normal geographical range is of concern given its relatively high pathogenicity and propensity to develop macrocyclic lactone resistance.

\section{Methods}

\section{Sample population}

The cow-calf operations participating in this study were enrolled in the western Canadian Cow-Calf Surveillance Network (WCCCSN). The formation of this network has previously been described $[15,16]$. Briefly, producers for the WCCCSN were enrolled based on national agricultural census data to obtain a geographically representative sample population from western Canada, including Alberta, Saskatchewan, and Manitoba. Private veterinarians providing services to cow-calf operations in these provinces were asked to assist in recruiting producers. Inclusion criteria for participation were a minimum herd size of 100 cows, willingness to complete questionnaires, and allowing the collection of biological samples from cattle. In situations where more producers were identified than needed from a particular region, the required number of producers was selected on a random basis. At the time of data collection for this study, there were 111 cow-calf operations enrolled in the WCCCSN (55 in Alberta, 35 in Saskatchewan, and 21 in Manitoba). Through a survey on parasite management distributed in the summer of 2016, producers were asked to collect fecal samples from their heifers during the fall pregnancy diagnosis. 
They were also asked to voluntarily collect fecal samples from their 2016 calf crop around weaning (fall 2016 and winter 2017) and submit them to the laboratory for processing [17].

\section{Fecal sample collection}

Fecal sampling from heifers took place between September 2016 and February 2017. Each herd was provided with a sampling kit. The herd veterinarian was asked to collect fecal samples from the rectum of 20 randomly selected heifers based on the availability; if 20 heifers were not available, the youngest bred cows were sampled for a total of 20 samples per herd. This sampling strategy resulted in a median age of sampled heifers of 20 months (range 12-36 months). Calves were sampled from November 2016 to February 2017. Producers were instructed to collect at least two handfuls of feces (freshly voided or rectally collected) from 20 conveniently selected individual calves. The median age of calves sampled was eight months (range 7-9 months). The number of samples collected from each herd ranged from 6 to 20 samples (median 20) for heifers and 10 to 25 (median 20) for calves. Individual fecal samples from heifers and calves were collected into labeled plastic bags, with the air expelled, stored in an insulated container at room temperature, and shipped within 24 hours of collection to the laboratory at the University of Saskatchewan, Saskatoon, Saskatchewan, Canada (heifer samples), or the laboratory at the University of Calgary, Calgary, Alberta, Canada (calf samples).

\section{Fecal egg counting}

There were 1,655 heifer samples (Alberta $=876$, Saskatchewan $=468$, Manitoba $=311$ ) from 85 herds (Alberta $=45$, Saskatchewan $=24$, Manitoba $=16)$ available to obtain fecal egg counts $(F E C)$ and subsequent analysis. For the calves, 824 (Alberta $=447$, Saskatchewan $=211$, Manitoba $=166$ ) fecal samples from 42 herds (Alberta $=23$, Saskatchewan $=10$, Manitoba $=9$ ) were processed.

Individual fecal samples were processed within three to five days of collection. A modified Wisconsin sugar flotation technique with minor modifications was used to process fecal samples [18]. Five and $3 \mathrm{~g}$ of feces were used to obtain FEC from heifers and calves, respectively. Gastrointestinal nematode eggs were identified as strongyle-type spp., Nematodirus spp., or Trichuris spp.

\section{Coproculture larval harvesting}

A different pooling strategy was used for the heifer and calf samples because the low number of larvae harvested per herd for the former did not allow meaningful quantitative data to be generated at the individual herd level.

\section{Heifer samples}

A modified coproculture protocol was used to harvest third stage nematode larvae (L3) [19]. Briefly, a composite fecal sample was prepared for each herd by pooling $12 \mathrm{~g}$ of homogenized feces from the individual heifers and three coprocultures set up comprising eighty grams of the composite feces mixed with vermiculite and tap water in a $250 \mathrm{~mL}$ glass. Cultures were incubated at room temperature 
(approximately 20 to $23^{\circ} \mathrm{C}$ ) for 21 days, after which time L3 were harvested, washed twice in tap water by centrifugation at $3725 \mathrm{~g}$ for 3 minutes before resuspending in $0.3 \mathrm{~mL}$ of tap water, and fixed by the addition of $0.7 \mathrm{ml}$ of $95 \%$ ethanol. The larvae derived from the three coprocultures per herd composite fecal samples were then pooled to provide one pool of harvested larvae per herd. After the enumeration of an aliquot of larvae by microscopy, samples were stored in the refrigerator $\left(4^{\circ} \mathrm{C}\right)$ for several weeks before being sent to the University of Calgary laboratory and kept frozen at $-80^{\circ} \mathrm{C}$ for archiving and ITS-2 rDNA nemabiome metabarcoding. Since there were insufficient larvae obtained for meaningful quantitative analysis at the individual herd level, the L3 harvested from each herd were put into two pools per province; one pool for small herds ( $\leq 300$ cow-calf pairs) and one pool for large herds (> 300 cow-calf pairs). The small herd pools comprised 29, 16, and 10 herds, and the large herd pools comprised 15, 8 , and 5 herds for Alberta, Saskatchewan, and Manitoba, respectively. This pooling strategy was chosen to give some degree of replication within the limitations of the small number of larvae harvested from many individual herds and compare the species abundance between herds of different sizes and between provinces. The total number of $L 3$ in the small and large herd pools, respectively were: Alberta $=4,200$ and 3,900 L3, Saskatchewan $=3,600$ and 4,400 L3, Manitoba $=3,800$ and 5,200 L3. Three separate aliquots of $1000 \mathrm{~L} 3$ were then taken from each pool to prepare triplicate genomic DNA samples for nemabiome metabarcoding.

\section{Calf samples}

For calves, individual animal coprocultures were prepared from $40 \mathrm{~g}$ of homogenized feces as per the method of Roberts and O'Sullivan [19]. Culture conditions and harvesting procedures were similar to those described for the heifer samples. Larvae were counted by microscopy, and $50 \%$ of the larvae from individual samples were pooled to create a single herd-level pool of larvae. Pooled L3 were washed, centrifuged, enumerated, and fixed in $95 \%$ ethanol, similar to the procedure described for heifers. Samples were stored at $-80^{\circ} \mathrm{C}$ until being processed for ITS-2 rDNA nemabiome metabarcoding.

There were adequate L3 counts for quantifying GIN species proportions at the herd level for 40 out of 42 herds sampled ( $n=22,10$, and 8 ) in Alberta, Saskatchewan, and Manitoba, respectively). Genomic DNA prepared from 250 L3 from each herd-level pool was used for ITS-2 rDNA sequencing. There were sufficient larvae in most herd-level pools for two or three separate aliquots of $250 \mathrm{~L} 3$ to prepare duplicate or triplicate genomic DNA samples. However, four herd-level pools had insufficient larvae for duplicate samples; consequently, a single aliquot of 250 L3 was processed (Additional file: Figure 1).

\section{ITS-2 rDNA nemabiome metabarcoding}

The ITS-2 rDNA nemabiome metabarcoding methodology has been previously described and validated [9]. Protocol details are available at https://www.nemabiome.ca/sequencing.html [20]. Briefly, larvae were placed in a Proteinase $\mathrm{K}(120 \mu \mathrm{g} / \mathrm{mL})$ lysis buffer $(50 \mathrm{mM} \mathrm{KCl}, 10 \mathrm{mM}$ Tris (pH8.3), $2.5 \mathrm{mM}$ $\mathrm{MgCl}_{2}, 0.45 \%$ Nonidet P-40, $0.45 \%$ Tween $20,0.01 \%$ (w/v) gelatin) to create pooled crude lysates.

Molecular grade $\mathrm{ddH}_{2} \mathrm{O}$ was used to make 1:10 dilutions of the pooled crude lysates used as a template 
for first-round PCR amplification of the ITS-2 rDNA target as described in Avramenko et al. [9]. Following purification with AMPure XP Magnetic Beads (1X) (Beckman Coulter Inc., Indianapolis, Indiana, USA), Illumina indices and P5/P7 sequencing tags (Illumina inc., San Diego, California, USA) were added using limited cycle PCR amplification, and the final amplicon products purified using the same method as above. Approximately $50 \mathrm{ng}$ of amplicon were pooled from each sample to make up the master sequencing library, quantified using the KAPA qPCR Library Quantification Kit (KAPA Biosystems inc., Wilmington, Massachusetts, USA). The final concentration of the pooled library was $12.5 \mathrm{nM}$, with the addition of 25\% PhiX Control v3 (Illumina, FC-110-3001), and it was run on an Illumina MiSeq Desktop Sequencer using a 500-cycle pair-end reagent kit (MiSeq Reagent Kits v2, MS-103-2003). Utilizing the Mothur software package, a bioinformatic pipeline was used to assign nematode species identity to each sequenced read using previously described methods [3]. Further details of the pipeline are available at https://www.nemabiome.ca/analysis.html [21]. Sequence reads were multiplied by previously validated correction factors specific to individual GIN species [9]. The number of sequence reads mapping to each species reference sequence was divided by the total number of mapped reads per sample to determine the percentage species composition of each sample. The total sequence read number mapping to ITS-2 rDNA reference sequences for each sample ranged from 13,401 to 32,801 reads for heifer samples and 15,178 to 83,935 reads for calf samples.

\section{Data analyses}

At the individual animal level, the proportions of individual fecal samples ( $95 \%$ confidence interval $(\mathrm{Cl})$ ) positive for strongyle-type, Nematodirus spp., and Trichuris spp. were determined for calves and heifers based on identifying at least one GIN egg under the microscope. The overall arithmetic mean EPG $( \pm$ standard deviation (SD)) of the three morphologically different GIN egg types were also calculated for both heifers and calves. For calves, the herd-level arithmetic mean EPG ( \pm SD) of all GIN egg types was calculated and presented with the relative herd-level GIN species proportions.

At the provincial level, the arithmetic mean EPG $( \pm$ SD) of strongyle-type FEC for each province was calculated, and the statistical difference between provinces was determined using a Generalized Estimating Equations model with a negative binomial distribution and log link function, accounting for clustering at the herd-level for both calves and heifers. Nematodirus spp. and Trichuris spp. FEC were very low in both calves and heifers; therefore, their provincial-level arithmetic means were not estimated.

Alpha diversity was calculated to determine the overall species diversity of GIN populations in calves and heifers within a province. For calves, species diversity data of analytical replicates (i.e., 250 L3 aliquots; 62, 36, and 20 aliquotes for Alberta, Saskatchewan, and Manitoba, respectively) of herd-level pools were used for the mean comparison. For heifers, species diversity data of analytical replicates (i.e., 1000 L3 aliquots; 12 aliquots per province) of each provincial pool were used for the mean comparison. Analytical replicates for heifers were meaningful because they represent multiple herds within a province as larvae from many individual herds went into each provincial pool. The calculations were performed in Mothur v.1.36.1 using the built-in inverse Simpson calculation as previously reported [9]. To assess whether the 
inverse Simpson index differed significantly between each province, a one-way ANOVA, assuming nonequal variances, was performed using a Games-Howell post-hoc comparison in SPSS statistical software (IBM Corp. Released 2012. IBM SPSS Statistics for Macintosh, Version 21.0. Armonk, New York, USA).

Beta diversity estimation was performed for three major species, $O$. ostertagi, $C$. oncophora, and $C$. punctata, using the MetaStats plugin in 275 Mothur v. 1.36.1, using 1000 permutations and default parameters to determine whether the species composition differed in calves and heifers between two different provinces [22]. MetaStats assumed that the data was not normally distributed; therefore, modified non-parametric t-tests (two-tailed) for pairwise comparisons of beta diversity estimations of each GIN species in each province were used [22]. This method can overestimate the GIN species with a lower abundance; therefore, significance was not claimed if the species were present at less than $2 \%$ on average in both comparable groups. Significance was declared if $P<0.05$. In terms of calves, the arithmetic mean FEC of strongyle-type spp., Nematodirus spp., and Trichuris spp. for each herd were reported with the relative GIN species proportions in each sample.

\section{Results}

\section{Fecal egg count and ITS-2 rDNA nemabiome data for gastrointestinal nematodes in heifers}

Strongyle-type eggs were detected in $92.0 \%(95 \% \mathrm{Cl}=91.0-93.0)$ of individual heifer samples and $100 \%$ of herds with an overall mean FEC of 5.3 (SD = 7.7) EPG (range = 0-92). Nematodirus spp. eggs, were detected in $1.8 \%(95 \% \mathrm{Cl}=1.0-3.0)$ of individual samples and $24.7 \%(21 / 85)$ of herds with an overall mean FEC of 0.01 (SD $=0.1$ ) EPG (range $=0-2.4$ ). Trichuris spp. eggs were detected in $1 \%$ of individual samples $(95 \% \mathrm{Cl}=1.0-2.0)$ and $21.2 \%(18 / 85)$ of herds with an overall mean FEC of $0.003(\mathrm{SD}=0.3)$ EPG (range $=0-0.6)$. The mean strongyle-type FEC of heifers in Alberta, Saskatchewan, and Manitoba were $5.1(\mathrm{SD}=6.5$, range $=0-55.6), 5.9(\mathrm{SD}=8.7$, range $=0-92)$, and $5.2(\mathrm{SD}=9.3$, range $=0-85)$, respectively, and they were not significantly different (Wald $\chi^{2}=0.66$, number of observations $=1655$, number of groups $=85, P=0.719)$.

ITS-2 rDNA nemabiome metabarcoding revealed that 0 . ostertagi was the predominant parasite species in heifers from Alberta and Saskatchewan herds (Figure 1), with an overall relative abundance of 53.9 and $52.0 \%$ in Alberta small and large herds, respectively, and 59.3 and $61 \%$ in Saskatchewan small and large herds, respectively. Cooperia oncophora was the second most abundant GIN in those two provinces, making up 39.4 and $28.6 \%$ of the parasite species proportions overall from small and large herds in Alberta, respectively, and 23.5 and $34.9 \%$ of the GIN populations from small and large herds in Saskatchewan, respectively. In contrast, Cooperia punctata was the predominant GIN species in Manitoba, with an overall relative abundance of $51.5 \%$ and 54.8 in small and large herds, respectively. Ostertagia ostertagi was the second most abundant GIN in Manitoba's small and large herds, with an overall relative abundance of 22.9 and $28.4 \%$, followed by $C$. oncophora with an overall relative abundance of 10.0 and $10.2 \%$ in those herds, respectively. Other GIN species were present in smaller proportions in Alberta, Saskatchewan, and Manitoba regional pools, including Oesophagostomum 
radiatum (4.1-7.4\%), Haemonchus placei (0.9-2.4\%), Trichostrongylus longispicularis (0.1-1.6\%), Haemonchus contortus (0-0.2\%), and Orloffia bisonis (0-0.3\%).

The comparison of inverse Simpson index for alpha diversity manifested a significant difference in overall GIN species diversity between provinces $\left(F_{(2,33)}=27.3, P<0.001\right)$. The post-hoc comparisons of the inverse Simpson indexes revealed that the overall GIN species diversity in heifers was greater in Manitoba (Mean index $=3.60, \mathrm{SD}=0.64$ ) herds than the Alberta (Mean index $=2.54, \mathrm{SD}=0.32)$ herds $(P$ $=0.037)$. Similarly, the overall GIN species diversity of Manitoba herds was greater than Saskatchewan herds (Mean index $=2.31, \mathrm{SD}=0.36)$ herds $(P=0.009)$. Nevertheless, Alberta heifers' alpha GIN species diversity was not significantly different from Saskatchewan heifers $(P=0.765)$. Beta species diversity comparisons revealed that the relative abundance of $C$. punctata was significantly greater in heifers from Manitoba than Alberta $(t=35.1, P<0.001)$ and Saskatchewan $(t=33.6, P<0.001)$ (Table 1).

Gastrointestinal nematode populations in Alberta had a significantly greater abundance of $C$. oncophora $(t=12.1, P<0.001)$ and 0 . ostertagi $(t=27.5, P<0.001)$ than in Manitoba. Similarly, $C$. oncophora $(t=$ $10.0, P<0.001)$ and $O$. ostertagi $(t=26.7, P<0.001)$ abundances were significantly greater in Saskatchewan than Manitoba populations. Significant differences were not assessed for $N$. helvetianus, $O$. bisonis, $H$. placei, $O$. radiatum, and $T$. axei due to their low relative abundance $(<2 \%)$.

\section{Fecal egg count and ITS-2 rDNA nemabiome metabarcoding data for gastrointestinal nematodes in calves}

Strongyle-type eggs were detected in $91.1 \%(95 \% \mathrm{Cl}=89.0-93.0 \%)$ of individual calf samples and $100 \%$ of herds with an overall mean FEC of $17.9(\mathrm{SD}=20.3)$ (range $=0-178)$. Nematodirus spp. eggs, were detected in $30.7 \%(95 \% \mathrm{Cl}=27.6-34.0)$ of individual calf samples and $100 \%$ of herds with an overall mean FEC of $0.8(\mathrm{SD}=2.9)$ EPG (range = 0-36). Trichuris spp. eggs were detected in $33.1 \%(95 \% \mathrm{Cl}=$ $29.9-36.5)$ of individual calf samples and $85.7 \%(36 / 42)$ of herds with an overall mean FEC of 0.6 (SD = 2.0) $\mathrm{EPG}$ (range $=0-32 \mathrm{EPG}$ ). Mean strongyle-type FEC in calves from Alberta, Saskatchewan, and Manitoba were $15.3(\mathrm{SD}=15.8$, range $=0-88), 17.0(\mathrm{SD}=16.2$, range $=0-86)$, and $24.7(\mathrm{SD}=31.0$, range $=0-178)$, respectively, with no significant differences between those egg counts (Wald $\chi^{2}=2.27$, number of observations $=844$, number of groups $=42, P=0.322$ ).

ITS-2 rDNA nemabiome metabarcoding revealed that 0 . ostertagi was the most abundant species in Alberta and Saskatchewan, with a mean overall relative abundance of $50.8(S D=22.4$, range $=1.4-78.2)$ and $56.0 \%(S D=16.9$, range $=30.4-80.9)$, respectively. It was the predominant species in $18 / 22$ herds in Alberta and 7/10 herds in Saskatchewan (Figure 2). Cooperia oncophora was the second most abundant GIN species in those two provinces, with a mean overall abundance of 44.2 (SD $=21.3$, range $=21.5-$ 96.4 $)$ and $36.4 \%(S D=14.0$, range $=19.0-60.4)$ in Alberta and Saskatchewan, respectively. It was the predominant species in 4/22 Alberta herds and 2/10 Saskatchewan herds. In Alberta and Saskatchewan, C. punctata abundance was relatively low with a respective mean overall abundance of 0.16 (SD $=0.37$, range $=0-4.3)$ and $2.25 \%(S D=3.46$, range $=0.02-17.5)$. However, in Manitoba, $C$. punctata was the most abundant GIN species, with a mean overall relative abundance of $28.2 \%(S D=26.2$, range $=0.1-$ 
89.8). Cooperia punctata was the predominant species in $4 / 8$ of Manitoba herds. The mean relative species abundances of 0 . ostertagi and $C$. oncophora in Manitoba were 34.7\% (SD $=28.4$, range $=7.4-$ 82.2) and $31.9(S D=23.1$, range $=0.07-66.1)$, respectively. The former was the predominant species in $3 / 8$, and the latter was the predominant species in $1 / 8$ herds from Manitoba. Nematodirus helvetianus $(0.07-2.2 \%)$, O. bisonis $(0-0.7 \%)$, H. placei $(0.02-0.3 \%)$, O. radiatum $(0-0.2 \%)$, and T. axei $(0-0.07 \%)$ were also present in some herds, but their relative abundances were very small.

The comparisons of the inverse Simpson index revealed a significant difference in overall GIN species diversity between provinces $\left(F_{(2,116)}=8.59, P<0.001\right)$. The post-hoc comparisons of the inverse Simpson indexes indicated an overall lower GIN species diversity in the calves from Alberta (Mean index $=1.78, S D$ $=0.34$ ) herds than Manitoba (Mean index $=2.15, \mathrm{SD}=0.55)$ herds $(P=0.025)$. In parallel, the overall GIN species diversity in Alberta calves were lower than Saskatchewan (Mean index $=1.93, S D=0.20$ ) calves $(P=0.024)$. However, there was no difference in the overall species alpha diversity between the Manitoba and Saskatchewan GIN populations $(P=0.219)$. Beta diversity analysis manifested that Manitoba farms had a significantly greater abundance of $C$. punctata than Alberta $(t=4.79, P=0.001)$ and Saskatchewan $(t=4.41, P=0.001)$ (Table 2). The relative abundance of $C$. punctata was significantly lower in Alberta herds than in Saskatchewan herds $(t=-3.61, P=0.001)$. Gastrointestinal nematode populations in Alberta herds had a significantly greater abundance of $C$. oncophora than GIN populations found on farms from Manitoba ( $t=2.50, P=0.022)$ and Saskatchewan $(t=2.05, P=0.044)$. Furthermore, $O$. ostertagi was significantly lower in GIN populations found in Manitoba farms compared to GIN populations sampled in Alberta $(t=-2.40, P=0.023)$ and Saskatchewan farms $(t=-3.17, P=$ 0.003). Statistical differences were not assessed for $N$. helvetianus, $O$. bisonis, H. placei, O. radiatum, and T. axei due to their low relative abundance $(<2 \%)$.

\section{Discussion}

There is a lack of large-scale studies exploring regional differences of GIN species distribution and abundance in cattle, with most existing information being largely historical or anecdotal. This paucity of studies is partly because of the specialist expertise and time required for species identification and the lack of scalable tools for quantifying parasite species abundance from fecal samples. The recent development of ITS-2 rDNA nemabiome sequencing now provides a powerful new approach to investigate the relative abundance of GIN species [9]. A recent ITS-2 rDNA metabarcoding study of 50 beef herds across Canada determined that $O$. ostertagi and $C$. oncophora were the two most predominant GIN species overall, consistent with the general understanding that these two species are well adapted to cold temperate climates $[3,12]$. The only aspect of that study that suggested regional differences was the finding that $C$. punctata was present at a somewhat greater abundance overall in eastern Canada than in the west [3]. However, that species was still much less abundant than $O$. ostertagi and $C$. oncophora in the eastern Canadian herds overall. Here we have extended that work to explore potential regional differences in GIN species distribution and abundance in a larger scale study of cow-calf herds across three western Canadian provinces; Alberta (45 herds), Saskatchewan (24 herds), and Manitoba (16 herds). 
One of the challenges of working with cattle GIN in northern latitudes is the typically low fecal egg excretion and the consequent low recovery of eggs or larvae from fecal samples, particularly in adult cattle. Consequently, a pooling strategy was taken to provide a regional overview of the GIN species abundance in western Canadian beef operations in which samples from heifers were pooled at the provincial level (two pools per province based on herd size categorization), and samples from calves were pooled at the herd level. The ITS-2 rDNA nemabiome metabarcoding results were consistent with the previous study of Canadian beef calves finding that $O$. ostertagi was the predominant GIN species in both Alberta and Saskatchewan cow-calf operations, with the next most abundant species being $C$. oncophora [3]. However, for Manitoba, the results were markedly different from Alberta and Saskatchewan, with a very high abundance of $C$. punctata across multiple herds. This species was the most abundant GIN species in both the heifer and calf samples from Manitoba overall and was the single most abundant GIN species in calves from 4/8 herds sampled from Manitoba. This finding was surprising because $C$. punctata is considered poorly adapted to cooler climates and has only been reported to occur at high abundance at more southerly latitudes such as mid-west, southern US, and southern America, where it commonly dominates GIN communities $[3,8]$.

The high abundance of $C$. punctata at such a northerly latitude is concerning since this species is significantly more pathogenic than $C$. oncophora, the species of Cooperia that generally predominates in cattle in cooler temperate regions such as western Canada. Research in experimentally infected beef calves shows that $C$. punctata negatively impacts appetite and nutrient utilization resulting in significant reductions in average daily gain and dry matter intake [23]. The lack of historical data and detailed regional studies of GIN abundance in North American beef herds over the last few decades means it is not certain whether the high abundance of $C$. punctata in Manitoba represents a change and, if so, how recently this might have occurred. Nevertheless, this parasite species has not been frequently reported at such northerly latitudes in the past, and to our knowledge, never as the most predominant GIN species. Therefore, there is a strong possibility that these results reflect a range expansion of this species due to several factors. Trichostrongylid GIN are potentially sensitive to climate change since the part of the life cycle outside the host is very temperature- and moisture-dependent [24]. Range expansion associated with climate change has been suggested for several parasitic nematode species in the strongylid group. For example, the ruminant GIN nematode Haemonchus contortus is currently much more common in western Canadian and UK sheep farms than in previous decades, and modeling studies suggest that significant range expansion is likely to occur in northern Europe in the coming decades [25,26]. A major concern is the lengthening of the grazing season predicted in western Canada associated with global warming, which could lead to range expansion of GIN more usually found in southern latitudes. Changes in cattle GIN parasite distribution could also be associated with the selection pressure for routinely used anthelmintics, particularly macrocyclic lactones. Ivermectin resistance is now widespread in Cooperia spp. in North America, and a study in the USA in 2015 suggested that the overall prevalence of Cooperia spp. was increasing due to the selection pressure for macrocyclic lactone resistance $[8,14]$. Whatever the explanation of the unexpectedly high relative abundance of $C$. punctata in Manitoba cow-calf herds, its regional distribution in western Canada is not simply related to the latitude (Figure 3 ). The localized 
distribution in Manitoba may suggest a single or small number of introduction events into that region, perhaps associated with the importation of cattle from particular sources further south. It will be interesting to map the distribution of $C$. punctata in Manitoba in more detail and investigate potential risk factors for its presence and the history of animal movements and importations in the region. It is also noteworthy that the sampling timing was not markedly different between provinces and so not the reason for the differences in the GIN community composition. However, all samples from heifers and calves were collected in the fall and winter. Therefore, a more detailed temporal study is warranted both across the grazing season and between years to understand further the relative abundance and the regional distribution of this parasite.

Cooperia punctata is also one GIN species that most commonly develop resistance to macrocyclic lactones, with several USA cattle reports $[27,28]$. Ivermectin is currently the most widely used macrocyclic lactone in western Canadian beef operations [17]. We have recently confirmed ivermectin resistance in $C$. oncophora, $H$. placei, and $C$. punctata and provided evidence for ivermectin resistance of hypobiotic larvae of $O$. ostertagi in western Canada using integrated ITS-2 rDNA nemabiome metabarcoding and FEC reduction test results [29]. This finding suggests the emergence of ivermectin resistance in multiple GIN species in western Canada, including $C$. punctata.

\section{Conclusion}

In summary, this study has revealed striking regional differences in GIN species abundance in western Canada. Specifically, a previously unidentified high relative abundance of $C$. punctata in beef cattle in Manitoba. The lack of previous reports of this parasite species at such a northerly latitude raises the possibility that its range is geographically expanding in North America, perhaps under the influence of climate change, anthelmintic use, animal movement, and/or other as yet undetermined management factors. The emergence of $C$. punctata as a major constituent of cattle parasite communities in northern latitudes is potentially problematic given the high pathogenicity and capacity for anthelmintic resistance in this GIN species. This study also provides an excellent illustration of the value of ITS-2 rDNA nemabiome metabarcoding as a surveillance tool for ruminant GIN parasites.

\section{Abbreviations}

Cl: Confidence interval; EPG: Eggs per gram of feces; FEC : Fecal egg count/s; GIN: Gastrointestinal nematode/s; L3: Third stage nematode larvae; SD: Standard deviation; WCCCSN: Western Canadian Cow-Calf Surveillance Network

\section{Declarations}

\section{Ethics approval and consent to participate}


The animal care committee of the University of Saskatchewan approved this study (Protocol number: AUP 2014003). Prior consent was obtained from the herd owners regarding their participation in the study and biological sample collection from their cattle.

\section{Consent for publication}

Not applicable

\section{Availability of data and materials}

The datasets used and/or analyzed during the current study are available from the corresponding author on reasonable request.

\section{Competing interests}

The authors declare that they have no competing interests.

\section{Funding}

This study was funded by the Agriculture Development Fund (ADF; grant number 20150268) of the Government of Saskatchewan, the University of Saskatchewan, the Beef Cattle Research Council (BCRC; grant numbers ANH.04.17 and ANH.23.13), and Natural Sciences and Engineering Research Council (NSERC; grant number RGPIN-2015-03976) of Canada.

\section{Authors' contributions}

JRC, FDU, and JSG conceived and supervised the study. All authors were involved in study management, data interpretation, and manuscript review. EDS and FKW conducted the calf and heifer data collection and analyses. EDS, JSG, and FDU prepared the manuscript. EMR performed the calf fecal egg counts and, together with RWA, conducted all nemabiome metabarcoding. CDQ did the alpha and beta diversity analysis for the nemabiome data. CLW And SEP assisted with the statistical analyses. All authors read and approved the final manuscript.

\section{Acknowledgments}

The authors acknowledge Dr. Lianne McLeod for preparing the herd location map.

\section{References}

1. Myers GH, Taylor RF. Ostertagiasis in cattle. J Vet Diagn Invest. 1989;1:195-200.

2. Hawkins JA. Economic benefits of parasite control in cattle. Vet Parasitol. 1993;46:159-73.

3. Avramenko RW, Redman EM, Lewis R, Bichuette MA, Palmeira BM, Yazwinski TA, et al. The use of nemabiome metabarcoding to explore gastrointestinal nematode species diversity and anthelmintic treatment effectiveness in beef calves. Int J Parasitol. 2017;47:893-902. 
4. Bransby DI. Effects of grazing management practices on parasite load and weight gain of beef cattle. Vet Parasitol. 1993;46:215-21.

5. Stromberg BE, Averbeck GA. The role of parasite epidemiology in the management of grazing cattle. Int J Parasitol. 1999;29:33-9.

6. Fox NJ, Marion G, Davidson RS, White PCL, Hutchings MR. Climate-driven tipping-points could lead to sudden, high-intensity parasite outbreaks. R Soc Open Sci. 2015;2:140296.

7. Gethings OJ, Rose H, Mitchell S, Dijk JV, Morgan ER. Asynchrony in host and parasite phenology may decrease disease risk in livestock under climate warming: Nematodirus battus in lambs as a case study. Parasitology. 2015;142:1306-17.

8. Stromberg BE, Gasbarre LC, Ballweber LR, Dargatz DA, Rodriguez JM, Kopral CA, et al. Prevalence of internal parasites in beef cows in the United States: Results of the national animal health monitoring system's (NAHMS) beef study, 2007-2008. Can J Vet Res. 2015;79:290-5.

9. Avramenko RW, Redman EM, Lewis R, Yazwinski TA, Wasmuth JD, Gilleard JS. Exploring the gastrointestinal "Nemabiome": deep amplicon sequencing to quantify the species composition of parasitic nematode communities. PLOS ONE. 2015;10:e0143559.

10. Avramenko RW, Redman EM, Melville L, Bartley Y, Wit J, Queiroz C, et al. Deep amplicon sequencing as a powerful new tool to screen for sequence polymorphisms associated with anthelmintic resistance in parasitic nematode populations. Int J Parasitol. 2019;49:13-26.

11. Redman E, Queiroz C, Bartley DJ, Levy M, Avramenko RW, Gilleard JS. Validation of ITS-2 rDNA nemabiome sequencing for ovine gastrointestinal nematodes and its application to a large scale survey of UK sheep farms. Vet Parasitol. 2019;275:108933.

12. Hildreth MB, McKenzie JB. Epidemiology and control of gastrointestinal nematodes of cattle in northern climates. Vet Clin North Am Food Anim Pract. 2020;36:59-71.

13. Fox MT, Gerrelli D, Pitt SR, Jacobs DE, Gill M, Gale DL. Ostertagia ostertagi infection in the calf: effects of a trickle challenge on appetite, digestibility, rate of passage of digesta and liveweight gain. Res Vet Sci. 1989;47:294-8.

14. Kaplan RM. Biology, epidemiology, diagnosis, and management of anthelmintic resistance in gastrointestinal nematodes of livestock. Vet Clin North Am Food Anim Pract. 2020;36:17-30.

15. Moggy M, Pajor E, Thurston W, Parker S, Greter A, Schwartzkopf-Genswein K, et al. Attitudes of western Canadian cow-calf producers towards the code of practice for the care and handling of beef cattle. Can Vet J. 2017;58:1209-11.

16. Waldner CL, Parker S, Gesy KM, Waugh T, Lanigan E, Campbell JR. Application of direct polymerase chain reaction assays for Campylobacter fetus subsp. venerealis and Tritrichomonas foetus to screen preputial samples from breeding bulls in cow-calf herds in western Canada. Can J Vet Res. 2017;81:91-9.

17. Wills F, Campbell J, Parker S, Waldner C, Uehlinger F. Gastrointestinal nematode management in western Canadian cow-calf herds. Can Vet J. 2020;61:382-8. 
18. Ito S. Modified Wisconsin sugar centrifugal-flotation technique for nematode eggs in bovine feces. $J$ Jpn Vet Med Assoc. 1980;33:424-9.

19. Roberts FHS, O'Sullivan PJ. Methods for egg counts and larval cultures for strongyles infesting the gastrointestinal tract of cattle. Aust J Agric Res. 1950;1:99-102.

20. Nemabiome. https://www.nemabiome.ca/sequencing.html. Accessed 20 May 2021

21. Nemabiome. https://www.nemabiome.ca/analysis.html. Accessed 20 May 2021

22. White JR, Nagarajan N, Pop M. Statistical methods for detecting differentially abundant features in clinical metagenomic samples. PLOS Comput Biol. 2009;5:e1000352.

23. Stromberg BE, Gasbarre LC, Waite A, Bechtol DT, Brown MS, Robinson NA, et al. Cooperia punctata: Effect on cattle productivity? Vet Parasitol. 2012;183:284-91.

24. Aleuy OA, Kutz S. Adaptations, life-history traits and ecological mechanisms of parasites to survive extremes and environmental unpredictability in the face of climate change. Int $\mathrm{J}$ Parasitol Parasites Wildl. 2020;12:308-17.

25. Queiroz C, Levy M, Avramenko R, Redman E, Kearns K, Swain L, et al. The use of ITS-2 rDNA nemabiome metabarcoding to enhance anthelmintic resistance diagnosis and surveillance of ovine gastrointestinal nematodes. Int J Parasitol Drugs Drug Resist. 2020;14:105-17.

26. Rose H, Caminade C, Bolajoko MB, Phelan P, Dijk J van, Baylis M, et al. Climate-driven changes to the spatio-temporal distribution of the parasitic nematode, Haemonchus contortus, in sheep in Europe. Glob Change Biol. 2016;22:1271-85.

27. Gasbarre LC, Smith LL, Hoberg E, Pilitt PA. Further characterization of a cattle nematode population with demonstrated resistance to current anthelmintics. Vet Parasitol. 2009;166:275-80.

28. Gasbarre LC, Smith LL, Lichtenfels JR, Pilitt PA. The identification of cattle nematode parasites resistant to multiple classes of anthelmintics in a commercial cattle population in the US. Vet Parasitol. 2009;166:281-5.

29. De Seram E. Epidemiology and impact of gastrointestinal nematode infection in young beef cattle in western Canada [dissertation on the internet]. Saskatoon, Saskatchewan: University of Saskatchewan; 2021. Available from: https://harvest.usask.ca/handle/10388/13208

\section{Tables}

Table 1. Beta-diversity (MetaStats) significance for individual parasite species of gastrointestinal nematodes in provincial level larval pools from heifers between 12-36 months of age from 83 cow-calf operations in Alberta (AB; $n=44)$, Saskatchewan (SK; $n=24)$ and Manitoba (MB; $n=15)$, Canada. 


\begin{tabular}{lllllll} 
Nematode species & \multicolumn{5}{c}{ Mean $\%( \pm S D)^{\text {a }}$} & \multicolumn{5}{l}{ Statistical analysis } \\
\cline { 2 - 7 } & AB & SK & MB & AB vs SK & AB vs MB & SK vs MB \\
\hline Ostertagia ostertagi & 53.6 & 60.0 & 24.8 & $t=5.86$ & $t=27.5$ & $t=26.7$ \\
& $(1.8)$ & $(3.3)$ & $(3.2)$ & $P<0.001^{*}$ & $P<0.001^{*}$ & $P<0.001^{*}$ \\
\hline Cooperia oncophora & 32.7 & 29.3 & 9.6 & $t=1.35$ & $t=12.1$ & $t=10.0$ \\
& $(6.1)$ & $(6.3)$ & $(2.5)$ & $P=0.190$ & $P<0.001^{*}$ & $P<0.001^{*}$ \\
\hline Cooperia punctata & 4.0 & 3.5 & 54.0 & $t=0.589$ & $t=35.1$ & $t=33.6$ \\
& $(1.7)$ & $(2.4)$ & $(4.6)$ & $P=0.561$ & $P<0.001^{*}$ & $P<0.001^{*}$
\end{tabular}

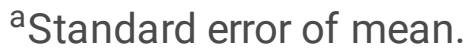

*Statistically significant.

Table 2. Beta-diversity (MetaStats) significance for individual parasite species of gastrointestinal nematodes in herd level larval pools from calves $<1$ year of age from 40 cow-calf operations in Alberta $(A B ; n=22)$, Saskatchewan $(S K ; n=10)$ and Manitoba $(M B ; n=8)$, Canada.

\begin{tabular}{lllllll} 
Nematode species & \multicolumn{3}{c}{ Mean $\%( \pm \mathrm{SD})^{\mathrm{a}}$} & \multicolumn{4}{c}{ Statistical analysis } \\
\cline { 2 - 7 } & AB & SK & MB & AB vs SK & AB vs MB & SK vs MB \\
\hline Ostertagia ostertagi & 50.8 & 56.0 & 34.7 & $t=1.29$ & $t=2.40$ & $t=3.17$ \\
& $(22.4)$ & $(16.9)$ & $(27.2)$ & $P=0.188$ & $P=0.023^{*}$ & $P=0.003^{*}$ \\
\hline Cooperia oncophora & 44.2 & 36.4 & 31.9 & $t=2.05$ & $t=2.50$ & $t=0.959$ \\
& $(21.3)$ & $(14.0)$ & $(23.1)$ & $P=0.044^{*}$ & $P=0.022^{*}$ & $P=0.352$ \\
\hline Cooperia punctata & 0.16 & 2.3 & 28.2 & $t=3.61$ & $t=4.79$ & $t=4.41$ \\
& $(0.94)$ & $(3.5)$ & $(26.2)$ & $P=0.001^{*}$ & $P=0.001^{*}$ & $P=0.001^{*}$
\end{tabular}

aStandard deviation.

*Statistically significant.

\section{Figures}




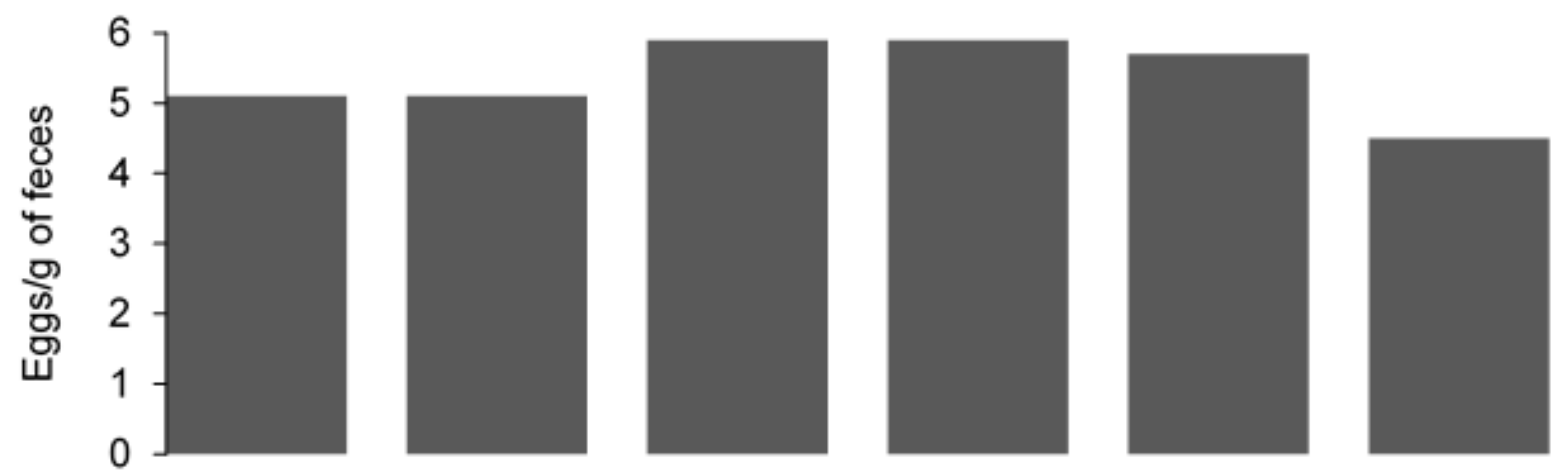

b

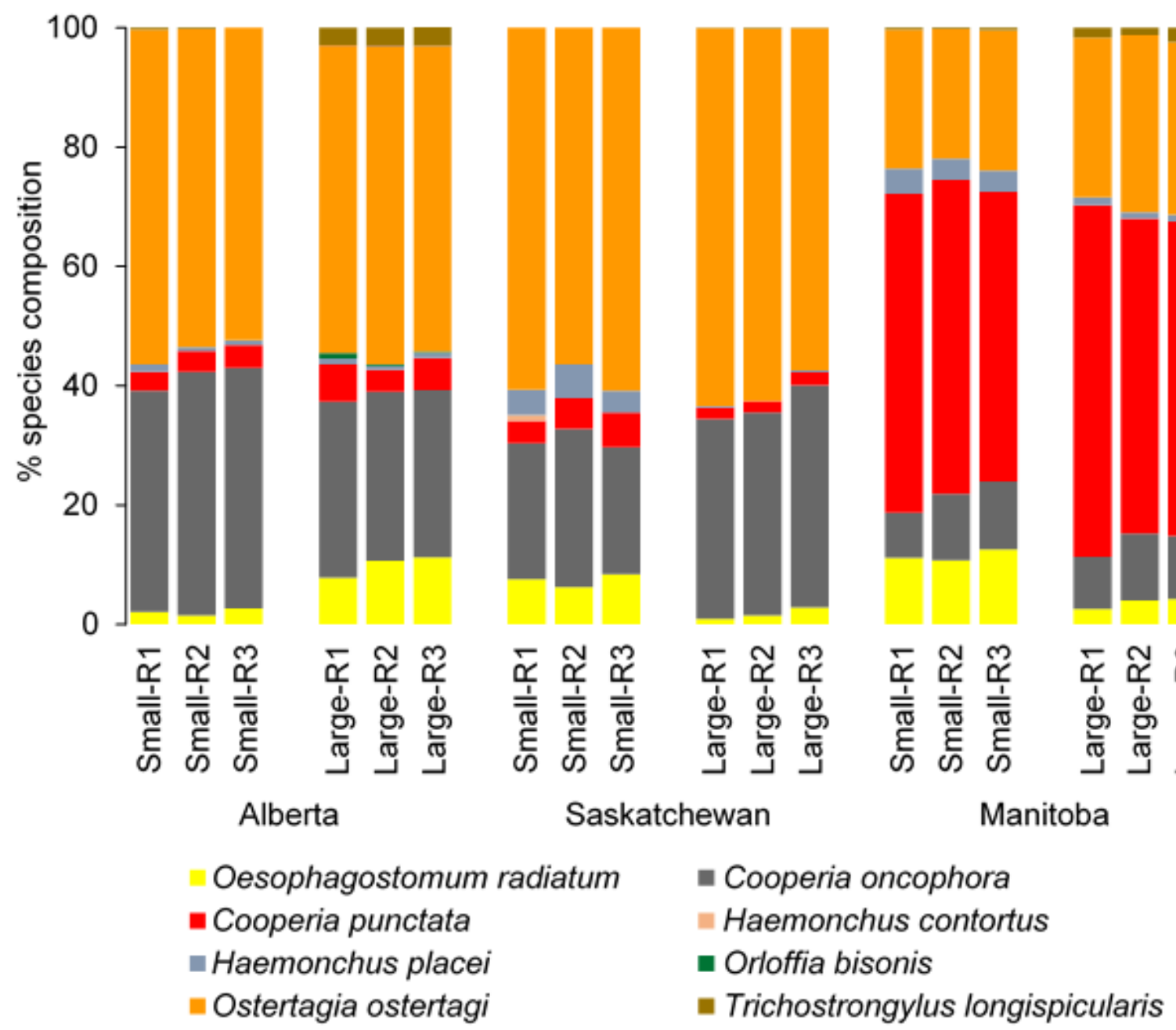

Figure 1

Fecal egg counts and gastrointestinal nematode species proportions from heifer fecal sample pools from western Canadian beef farms. Samples were pooled at the provincial level with two pools per province categorized based on herd size (small $\leq 300$ cow-calf pairs, large $>300$ cow-calf pairs). Three independent aliquots (R1, R2, R3) of 1000 third stage larvae were taken for ITS-2 rDNA nemabiome metabarcoding for each pool. Figure A represents the arithmetic mean of individual animal strongyle-type 
fecal egg counts of each provincial pool. Figure B indicates the relative gastrointestinal nematode species proportions determined by ITS-2 rDNA nemabiome metabarcoding of third-stage larvae pooled by herd size and province.

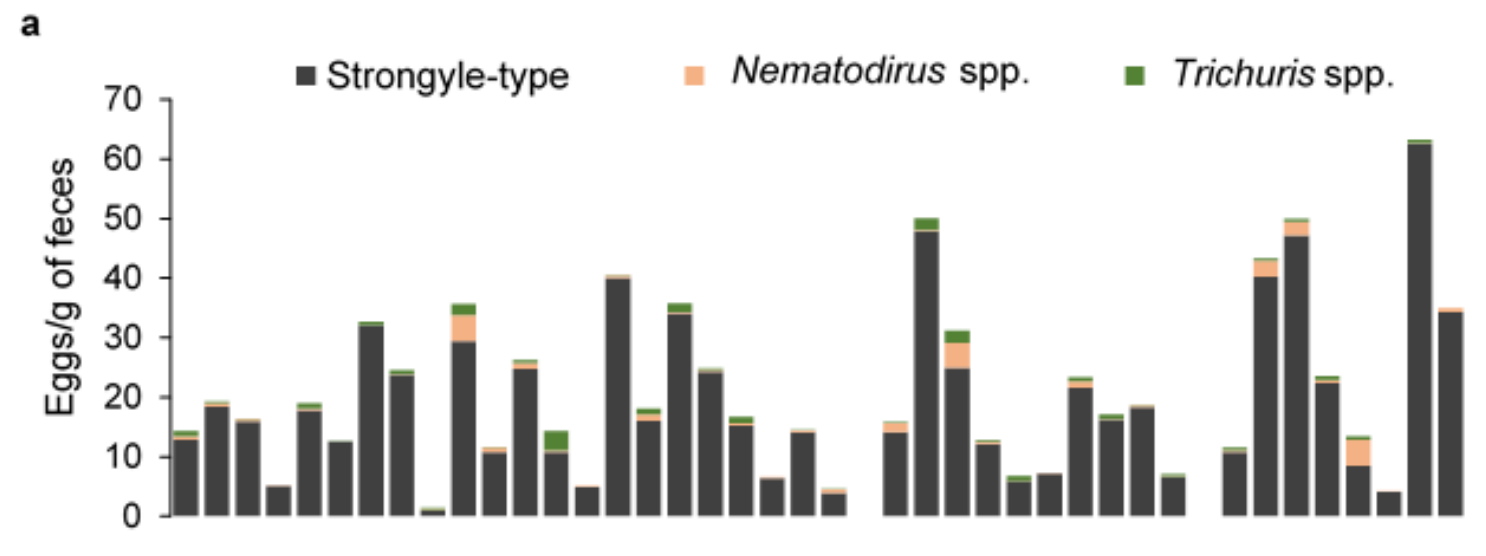

b

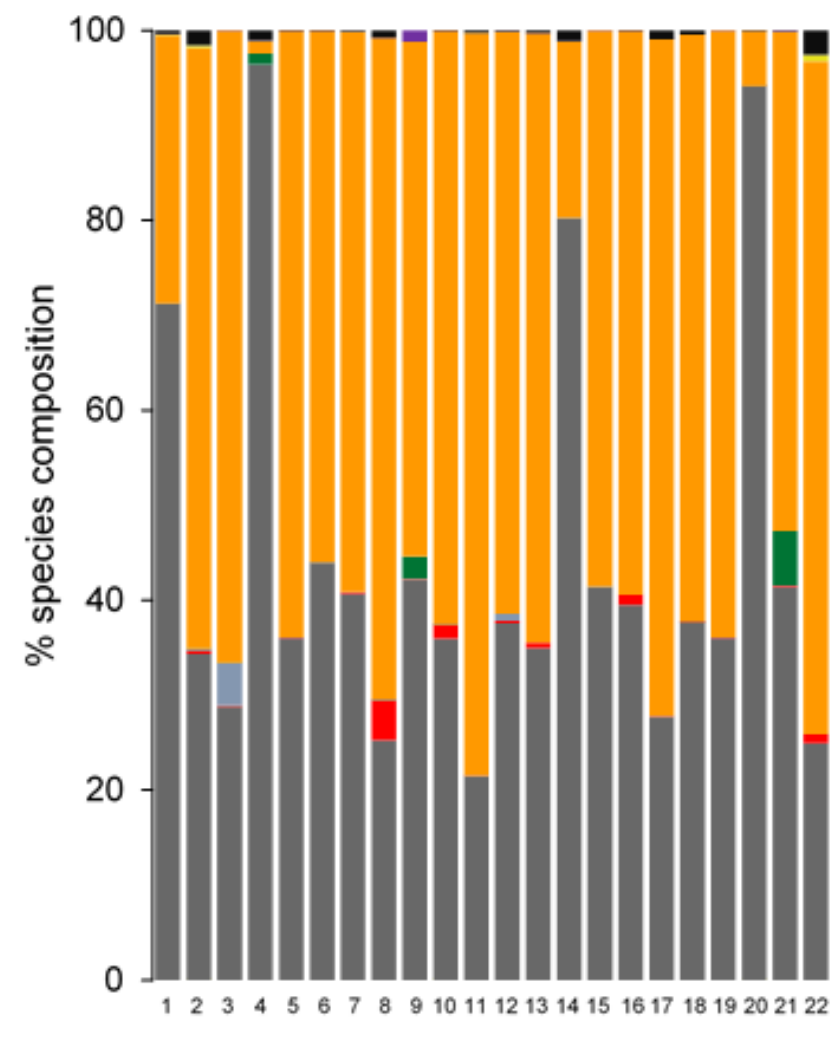

Alberta

Oesophagostomum radiatum

- Cooperia punctata

- Orloffia bisonis

Nematodirus helvetianus

- Trichostrongylus spp. unclassified

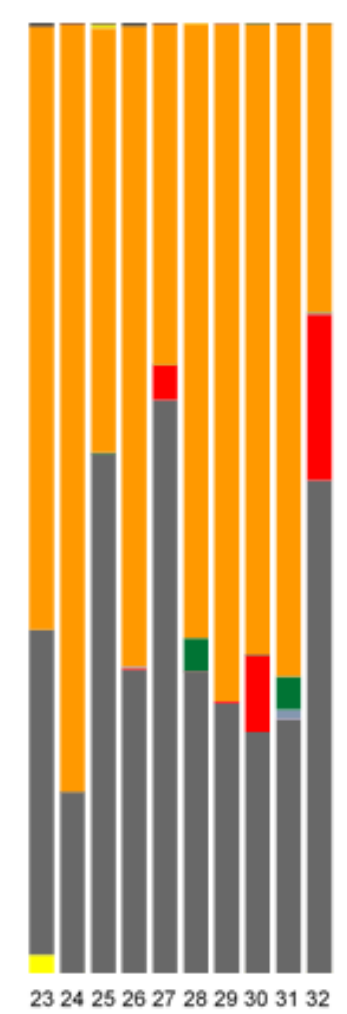

Saskatchewan

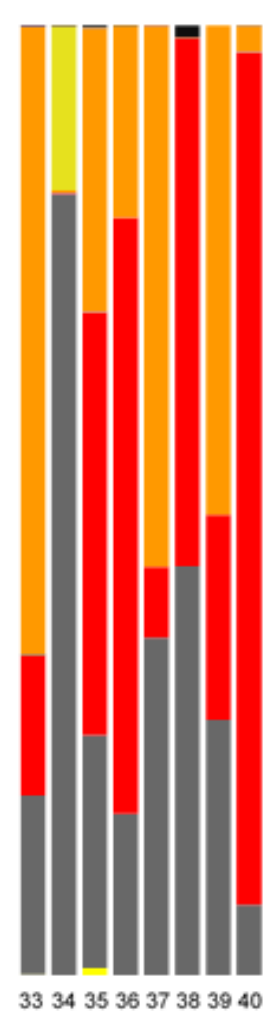

Manitoba

- Cooperia oncophora

- Haemonchus placei

= Ostertagia ostertagi

- Trichostrongylus axei

Figure 2

Fecal egg counts and gastrointestinal nematode species proportions in fecal samples of calves from 40 western Canadian cow-calf operations. Fecal samples from 10-20 individual calves were pooled at the 
herd level. Figure A represents the arithmetic means of 10-20 individual calf fecal egg counts of strongyle-type, Nematodirus spp. and Trichuris spp. eggs in each herd. Figure B represents the GIN species proportions in each determined by ITS-2 rDNA nemabiome metabarcoding of herd-level thirdstage larval pools. Numbers from 1-40 on the X-axis identify the individual herds. Except for herds 9, 14, 25 , and 34 , nemabiome metabarcoding was undertaken on duplicate or triplicate aliquots of 250 larvae per herd (Additional file 1: Figure 1).

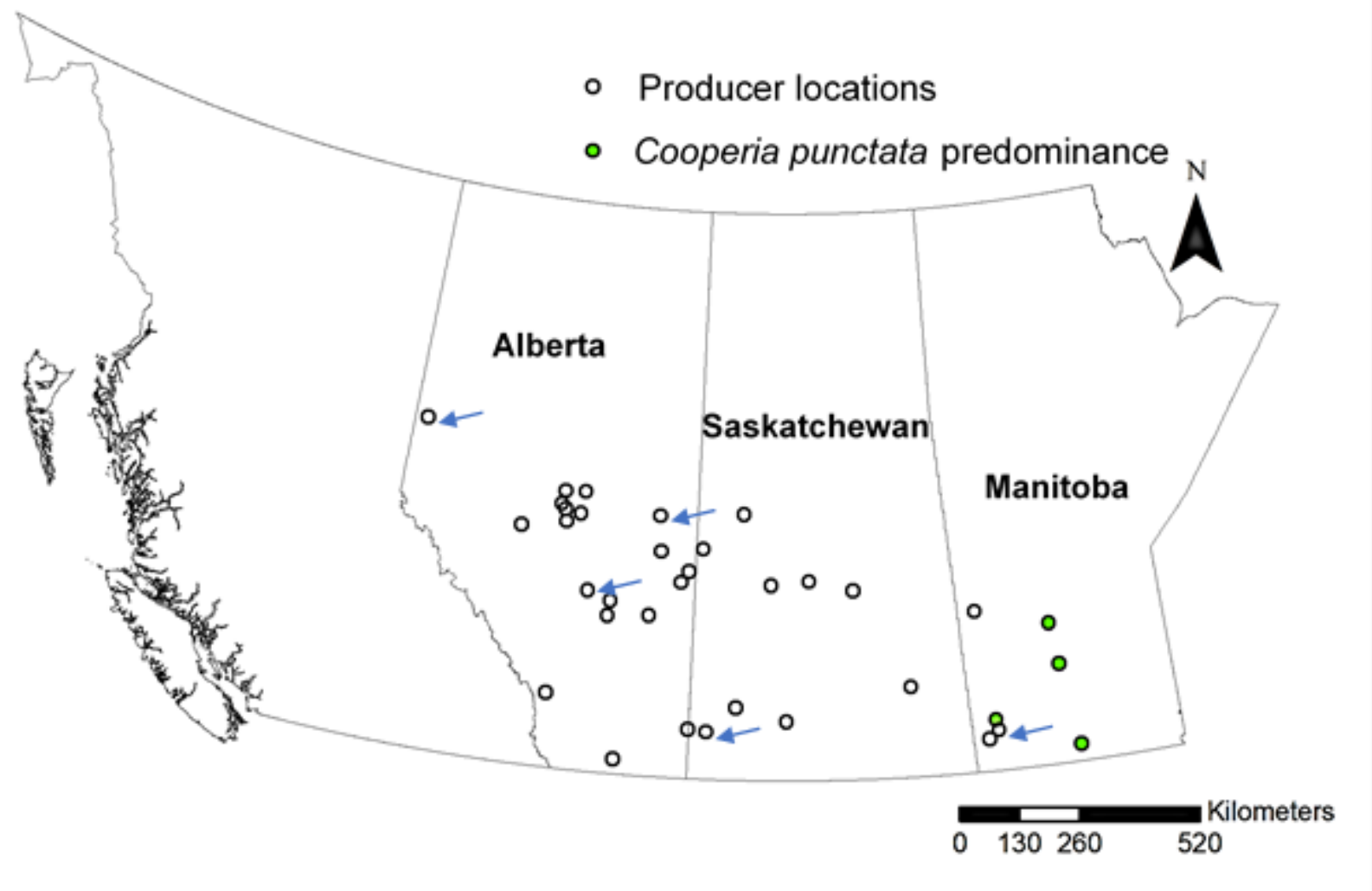

Figure 3

Approximate locations of 40 cow-calf operations where ITS-2 rDNA nemabiome metabarcoding data in fecal samples were available for calves. Those herds with a predominance of Cooperia punctata are indicated in green. Each location identified by an arrow contains two farms with the same postal code.

\section{Supplementary Files}

This is a list of supplementary files associated with this preprint. Click to download.

- C.punctatapredominanceadditionalfile1.xlsx

- C.punctatapredominancegraphicalabstract.tiff 